\title{
CORPUS Corpus
}

Archivos virtuales de la alteridad americana

Vol. 7, No 1 | 2017

Enero / Junio 2017

\section{El Padre João Pedro Gay (1815-1891) y su contribución a la historia social del guaraní de Corrientes}

Father João Pedro Gay and his Contribution to the Social History of Guarani in Corrientes

Franz Obermeier and Leonardo Cerno

OpenEdition

Journals

Electronic version

URL: http://journals.openedition.org/corpusarchivos/1774

DOI: $10.4000 /$ corpusarchivos. 1774

ISSN: $1853-8037$

Publisher

Diego Escolar

\section{Electronic reference}

Franz Obermeier y Leonardo Cerno, «El Padre João Pedro Gay (1815-1891) y su contribución a la historia social del guaraní de Corrientes », Corpus [En línea], Vol. 7, No 1 | 2017, Publicado el 29 junio 2017, consultado el 19 abril 2019. URL : http://journals.openedition.org/corpusarchivos/1774 ; DOI : 10.4000/corpusarchivos. 1774

This text was automatically generated on 19 April 2019

Licencia Creative Commons: Atribución-NoComercial 2.5 Argentina (CC BY-NC 2.5 AR) 


\section{El Padre João Pedro Gay (1815-1891) y su contribución a la historia social del guaraní de Corrientes}

Father João Pedro Gay and his Contribution to the Social History of Guarani in Corrientes

Franz Obermeier and Leonardo Cerno

\section{Introducción}

1 De mano del sacerdote francés Jean-Pierre (João Pedro) Gay conocemos hoy la copia de un poema en guaraní publicado en un periódico de Corrientes en 1867. La importancia del documento puede evaluarse en dos planos. En un plano biográfico constituye una muestra del interés del Padre Gay por la diversidad lingüística regional. Gay es actualmente conocido como historiador y su trabajo como lingüista, si bien existente, es prácticamente desconocido y quiere ponerse de relieve aquí. En un plano histórico, el aludido documento se presenta como uno de los pocos testimonios de la presencia del guaraní en la sociedad correntina de segunda mitad del siglo XIX. Este hecho, que merece una interpretación en sí misma, con independencia del plano biográfico, nos lleva a dividir este trabajo en dos partes: la primera orientada a rescatar la figura de Gay en tanto investigador de las lenguas de la región, y la segunda dedicada al análisis de este texto que constituye parte de su legado.

\section{João Pedro Gay y su mundo}

2 Nació en 1815 en Châteauroux (Francia, departamento de Hautes Alpes), ${ }^{1}$ y asistió al seminario de Gap. Llegó en 1842 a Montevideo, en 1843 a Santa Catarina (probablemente a Santa Ana de Vila Nova), y en 1844 a Río de Janeiro. En esta ciudad trabajó como profesor de francés y de matemáticas en el Colegio Saraiva. En 1848 fue cura en Alegrete (Rio Grande do Sul) y pidió la ciudadanía brasileña. En 1850 lo encontramos en San Borja, en el 
año 1861 recibió el título honorífico de Cônego Honorário da Capela Imperial. En junio 1865, durante la Guerra de la Triple Alianza, el teniente coronel Antonio Estigarribia destruyó su biblioteca y Gay se retiró a Uruguayana, 180 kilómetros al sur, sobre la costa del río Uruguay y frente a la provincia argentina de Corrientes. A partir de 1874 fue vicario en aquella ciudad, donde murió en 1891.

El personaje de João Pedro Gay es uno de los más interesantes de la época. Fue una de las piedras angulares de la investigación sobre los guaraníes en Brasil en el siglo XIX y tuvo muchos contactos en la corte de Pedro II. Vivió varias décadas en la región de la antigua provincia jesuítica del Uruguay. Tuvo además a su alcance las fuentes documentales de la región así como a los propios pobladores y su memoria local. Los juicios sobre su obra más conocida, "História da República Jesuítica do Paraguay", ${ }^{2}$ son hoy bastante negativos. Por poner un ejemplo, Furlong se refiere a "tantas confusiones de cosas y personas como hay en la Historia" (subrayado nuestro) y a "almácigo de errores". ${ }^{3}$ No obstante es necesario admitir que a pesar de sus muchas equivocaciones fue él uno de los primeros en tratar el tema, sobre todo el de los jesuitas y su retiro de la zona del Guairá.

Su contribución mayor a la historiografía de la época fue su relato como testigo de la invasión paraguaya de la región fronteriza. Este trabajo, reconocido como documento de valor histórico, es su libro "Invasão paraguaya na fronteira brazileira do Uruguay: desde seu principio até seu fim (de 10 de junho a 18 de setembro de 1865)". ${ }^{4}$ Su interés por las lenguas y su labor como lingüista son muy poco conocidos. Sabemos que pudo leer la versión guaraní de la "Conquista Espiritual" de Ruiz de Montoya en un manuscrito que utilizó para sus trabajos históricos. ${ }^{5}$ De ello se infiere que tuvo competencia en la lengua. Con respecto a su labor como lingüista, solo uno de sus trabajos fue publicado, en este caso referido a la lengua kaingang. Se trata del "Petit Vocabulaire de la langue des Bougres Couronnés", de 1857, publicado por el Instituto Histórico e Geográfico Brasileiro (IHGB), en Río. Es este uno de los primeros documentos sobre esa lengua indígena de Rio Grande do Sul, en el pueblo Guarita, hoy Reserva Indígena de Guarita, en el noroeste de Rio Grande do Sul.6 "Bougre" era en la terminología de la época uno de los términos empleado para los kaingang. Gay muestra con este trabajo que estaba interesado en realizar un tipo de investigación que hoy equivaldría al trabajo de campo lingüístico. Actualmente el trabajo es reconocido como contribución a la investigación de esta lengua casi no documentada en aquel tiempo (D'Angelis 2003). ${ }^{7}$

5 Su interés por el guaraní, patentizado en el documento que presentamos aquí, se explica además por el hecho de tener a su disposición las fuentes documentales de la época. La pervivencia de la cultura guaraní reduccional en su región, junto con la vitalidad de esa lengua indígena entre los criollos, por otra parte, pudieron haber motivado su curiosidad científica. Es posible que en el trato diario con los habitantes de la región haya aprendido el guaraní que empleó después con la traducción de "Conquista Espiritual".

6 Su gran amigo fue Aimé Bonpland. Aimé Jacques Alexandre Bonpland (en realidad Goujaud), nació en 1773 en La Rochelle, y murió en 1858 en la estancia de Santa Ana, en Corrientes. La figura de Bonpland queda sin duda en la memoria colectiva como el gran compañero de Alexander von Humboldt durante sus viajes por América Latina, entre 1799 y 1804. Bonpland es también una de las figuras más notables en la investigación sobre la historia natural de Sudamérica en el siglo XIX.

7 Después de la caída de Napoleón, el naturalista regresó al Río de la Plata en 1816 y decidió vivir en la zona de las antiguas misiones para dedicarse al cultivo de la yerba mate. Las 
circunstancias políticas tuvieron sin embargo un impacto negativo en su vida. El dictador paraguayo José Gaspar Rodríguez de Francia (1766-1840), quien quiso monopolizar el cultivo del mate, invadió en 1821 su plantación y la destruyó. Bonpland y los indígenas que trabajaban con él fueron enviados como prisioneros a Asunción. A pesar de la intervención de su amigo Humboldt ante el gobierno paraguayo, y de los esfuerzos diplomáticos de los gobiernos brasileño y británico y de muchas actividades de particulares franceses, fue liberado recién en 1829. Desde 1831 vivió en San Borja, y a partir de 1838 en la mencionada estancia de Santa Ana, cerca de la actual ciudad de Paso de los Libres, donde el gobierno le había concedido tierras. Murió en 1858. Hoy el lugar es llamado Bonpland en su honor.

8 Gay fue amigo personal de Bonpland durante varias décadas y después de su muerte fue también el ejecutor de su testamento. En esa ocasión hizo mucho por salvar para Francia el material de la importante documentación que Bonpland tenía sobre historia natural. ${ }^{8}$

9 Bonpland fue la figura clave de los franceses en esa región y fue frecuentemente visitado por exploradores de ese país, que también vieron en Gay a un compatriota que podía recibirlos. Sabemos por varias descripciones de viajes de la época que Gay también fue anfitrión de otros viajeros europeos, sobre todo franceses, que pasaban por la región. Los más importantes fueron Demersay y Moussy.

10 Alfred de Demersay (1815-1891) fue médico, viajó entre 1844 y1847 al Río de la Plata por orden del gobierno francés para explorar el potencial económico de la región. La orden fue dada a comienzos de la década de 1840. Este período coincide con los proyectos del agente colonial francés John Lelong, que quería iniciar la emigración francesa a la región del Río de la Plata, ahora consolidada después de la agitación política de la independencia. El gobierno francés quería tener un punto de vista neutral sobre el país y mandó a Demersay. Entre 1844 y 1847 Demersay permaneció en la zona, incluyendo cuatro meses en Paraguay. Entre otras cosas, visitó a Aimé Bonpland en su casa de San Borja en 1845. El objetivo comercial de su viaje no excluía un interés por la cultura de la región, sobre todo por su pasado jesuita. Más tarde, en 1862, Demersay realizó investigaciones en los archivos de España y Portugal. De vuelta en Francia publicó su "Histoire physique, économique et politique du Paraguay et des établissements des Jésuites" (Demersay 1860-1865).

11 Otro viajero de la región fue Victor Martin de Moussy (1810-1869), también médico, natural de Moussy-le-Vieux (departamento de Seine-et-Marne). Para el doctorado en medicina escribió su disertación sobre el tifus. ${ }^{9}$ Desde 1841 estuvo en el Río de la Plata ${ }^{10} \mathrm{y}$ también se interesó por la historia de la zona. ${ }^{11}$ Más tarde escribiría un libro sobre la región en base a sus experiencias y a su investigación personal. ${ }^{12}$ Moussy quiso ver a Bonpland en Santa Ana en 1855, pero este no estaba presente y Gay le mostró la biblioteca del naturalista, la cual estuvo a su cargo incluso durante la vida de su amigo:

De là [Santa Ana, donde vivía Bonpland] je fus à San Borja où son ami, M. Gay, me donna l'hospitalité. Gay, le curé du lieu, d'origine française, était devenu l'homme de confiance de Bonpland, le dépositaire de ses papiers et de ses collections. Tout cela était rassemblé, dit notre voyageur [Moussy, a quien parafrasea Hamy], dans une chambre à part avec les restes de la pharmacie dont Bonpland s'était servi pendant tant d'années. Les manuscrits formaient une masse extrêmement volumineuse et parfaitement rangée, mais les insectes avaient commencé à les piquer. Un herbier était également très attaqué. M. Gay me pria de revoir tout cela; il eût fallu passer quinze jours à San Borja et mes journées étaient comptées. Nous nous contentâmes de faire secouer les ballots et de les ranger de nouveau dans un 
endroit bien sec. M. Gay attendait Bonpland auquel il avait écrit de venir le plus tôt qu'il le pourrait, pour prendre une résolution au sujet de sa maison qui tombait en ruines et qu'il s'efforçait vainement de restaurer; il voulait profiter de cette occasion, me disait-il, pour lui faire remporter tous ses manuscrits et ses herbiers qui sans cela se perdraient (Hamy 1906, p. LXXXV). ${ }^{13}$

Gay fue también anfitrión del médico alemán Robert Avé-Lallemant (1812-1884), quien pasó muchos años trabajando como médico en Río de Janeiro. En un segundo viaje a Brasil, Avé-Lallemant atravesó el país entero y escribió dos importantes libros de viaje sobre el norte y el sur del país. ${ }^{14}$ La imagen que nos deja el viajero alemán sobre Gay es la del "personaje más conocido de la región". Así lo presenta en la descripción de su viaje por Rio Grande do Sul:

Ich hatte vom Herrn Provinzialpräsidenten einen Brief an Jean Pierre Gay, Vicar von Sta.Borja, einen geborenen Franzosen, der unbedingt die bekannteste Persönlichkeit der ganzen Gegend ist. Auf das allerfeundlichste ward ich von ihm aufgenommen und einquartiert und ich beschloß, für einige Tage von seiner Güte Gebrauch zu machen. (Avé-Lallemant, 1859, T 1, p. 318, subrayado nuestro).

[Tenía yo una carta del señor presidente provincial a Jean Pierre Gay, vicario de San Borja, un francés que sin dudas es la personalidad más conocida de la región. Me recibió y hospedó con la mejor de las amabilidades y decidí quedarme unos días para aprovechar su talento. Avé-Lallemant, 1859, T 1, p. 318, subrayado nuestro, traducción nuestra]

13 Junto con Gay visitó Avé-Lallemant la casa semidestruida de Bonpland y después de algunos días continuó su viaje. Contactos de este tipo sirvieron ciertamente a Gay para mantenerse al tanto de la ciencia en Europa incluso viviendo en un lugar tan apartado de los centros culturales como fue San Borja en esa época.

\section{Un documento único de Gay sobre el guaraní de Corrientes}

Para contribuir al estado actual de la investigación sobre Gay y su labor lingüística queremos presentar de manera preliminar un documento que caracteriza su interés lingüístico, en este caso sobre el guaraní. El documento que hemos encontrado se conserva en copia de su mano, pero no fue escrito por Gay mismo. Se trata de un poema anónimo en guaraní publicado en el diario "Eco de Corrientes" en 1867. El texto, que en un primer momento supusimos original, resultó ser la reproducción de un poema publicado previamente en el periódico paraguayo Cabichuí, en el mismo año. Pese a todo, la publicación en Corrientes no deja de ser significativa, no solo porque agrega información a una época poco documentada del pasado sociolingüístico provincial, sino también porque ocurre en el contexto de la Guerra de la Triple Alianza, que posicionó al Paraguay como enemigo. Subrayamos además que la copia de Gay es el único testimonio de la publicación correntina pues el número correspondiente de "Eco de Corrientes" no consta en las colecciones de este diario que se han conservado. ${ }^{15} \mathrm{El}$ texto copiado por Gay, hallado por Franz Obermeier, coautor de este artículo, consta en la Biblioteca Nacional de Río de Janeiro con la signatura I-47,5,1. ${ }^{16}$ Centrémonos ahora en las implicancias del documento para la historia lingüística de la región.

Son varios los problemas que la aproximación al texto plantea. ¿Por qué se publica en Corrientes un texto en guaraní procedente del Paraguay? ¿Hubo más publicaciones de este tipo? ¿Qué significaciones y valores se asociaron al hecho? ¿Qué papel jugó la Guerra 
de la Triple Alianza en la aparición del poema? Para responder a estas cuestiones es necesario presentar el contexto social, político y lingüístico de la región. En segundo lugar brindaremos los detalles lingüísticos del documento.

\section{Contexto histórico del poema}

16 Corrientes fue un diario de interés regional que se publicó en la ciudad de Corrientes desde agosto de 1866 y hasta mayo de 1868. De la literatura especializada se infiere que su importancia fue menor en comparación con El Progreso, de tendencia liberal, y El Independiente, de ideología federal, periódicos de mayor resonancia y que recibieron más atención por parte de los historiadores (Cf. Baratta 2013, p. 88; Ramírez Braschi 2004, p. 48 y p. 61). Apareció dos veces por semana, aunque hay noticias divergentes en cuanto a su frecuencia de publicación y a su duración. ${ }^{17}$ Manuel Mantilla, en su La Bibliografía Periodística de la Provincia de Corrientes, solo menciona el título. Uno de sus colaboradores fue José Hernández (1834-1886), el autor del clásico El gaucho Martín Fierro de 1872.

Según la copia de Gay el poema fue publicado el 16 de agosto de 1867. Esta fecha cae dentro del período de la Guerra de la Triple Alianza (1864-1870), guerra que enfrentó al Paraguay con Argentina, Brasil y Uruguay y cuyo escenario de acciones militares estuvo muy cerca de la provincia de Corrientes, y hasta parcialmente dentro de su territorio. Por su tema y su género el texto constituye un panegírico político: en cinco estrofas de cuatro versos celebra el cumpleaños de Francisco Solano López. La alabanza a López constituye, como se entiende, un detalle de interés porque se publicaba en el país enemigo del caudillo paraguayo. Por otra parte, varias semanas antes, el 24 de julio del mismo año, fecha exacta del cumpleaños de López, el mismo texto había aparecido publicado en el periódico de guerra paraguayo Cabichuí (Año 1, n² 22, p. 4, cf. Escobar y Salerno 2016). Pese a todo, dado que Cabichuí, así como otros periódicos de guerra paraguayos, publicaron algunos poemas y canciones de tradición oral (Lustig 2006, p. 249), no puede asegurarse que la versión correntina proceda de esa fuente escrita. Vayamos a la transcripción de la copia que llega a nuestras manos (ver IMAGEN N ${ }^{\circ} 1$ e IMAGEN N ${ }^{\circ} 2$ ). Agregamos la numeración de los versos para facilitar las referencias:

Hymno en guarany, festejando os annos do Marechal F. S. Lopes,

no dia 24 de Julho de 1867

Traducção em hespanhol.

Ñande Rubichá guaçú santo ára Nel dia del Santo de

Yarohorĭ enterobé: nuestro jefe, alegremonos todos, y

Ha yaha ñahetú ipó vayámos á besarle la mano en

Co 24 de julio pe el 24 de julio.

5 Co ára Tubichaiteba Este dia tan grandioso

Con razon ya festejá, con razon lo festejámos,

Porque ǐpipe onacé baecué porque en él fué que nació

Ñande carai guaçu Mariscal nuestro gran señor Mariscal.

TupÂme ya yeruré A Dios pidamos, que

10 Tapiá ti mÂrÂmi siempre lo conserve à este nuestro

Co ñande carai peabé, señor, y que nunca se

Ani mÂrÂmô haçĭ. enferme

OguerútÂmô el ataque Ojalá! traigan el ataque

Este dia los cambá, los negros neste día del Santo

15 Ñande Rubichá Santo ára de nuestro gran jefe, para que

ya rohorĭ porá haguá todos lo festejemos alegres. 
Y pahapé, paraguaios, Y al fin, paraguayos, Yahé en altas voces: digamos en altas voces: !Viva nuestro Mariscal !Viva nuestro Mariscal 20 Don Francisco Solano Lopes! Don Francisco Solano Lopes! Extrahido del Eco de Corrientes, viernes 16 de Agosto de $1867, \mathrm{n}^{\circ} 102$, pelo conego João Pedro Gay

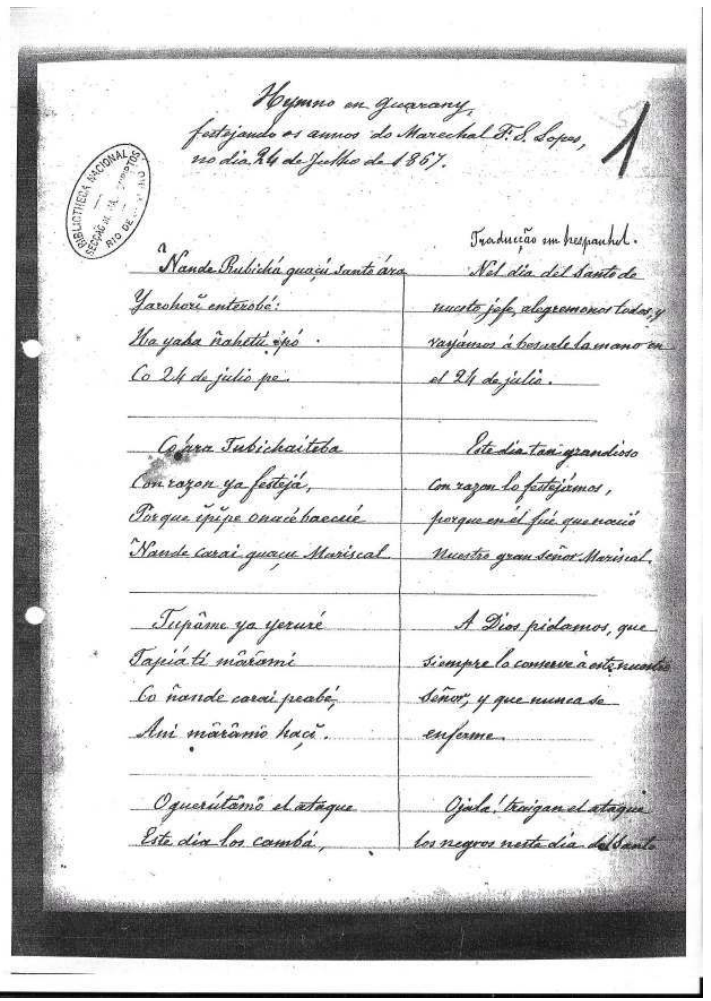

Imagen $\mathrm{N}^{\circ} 1$ 


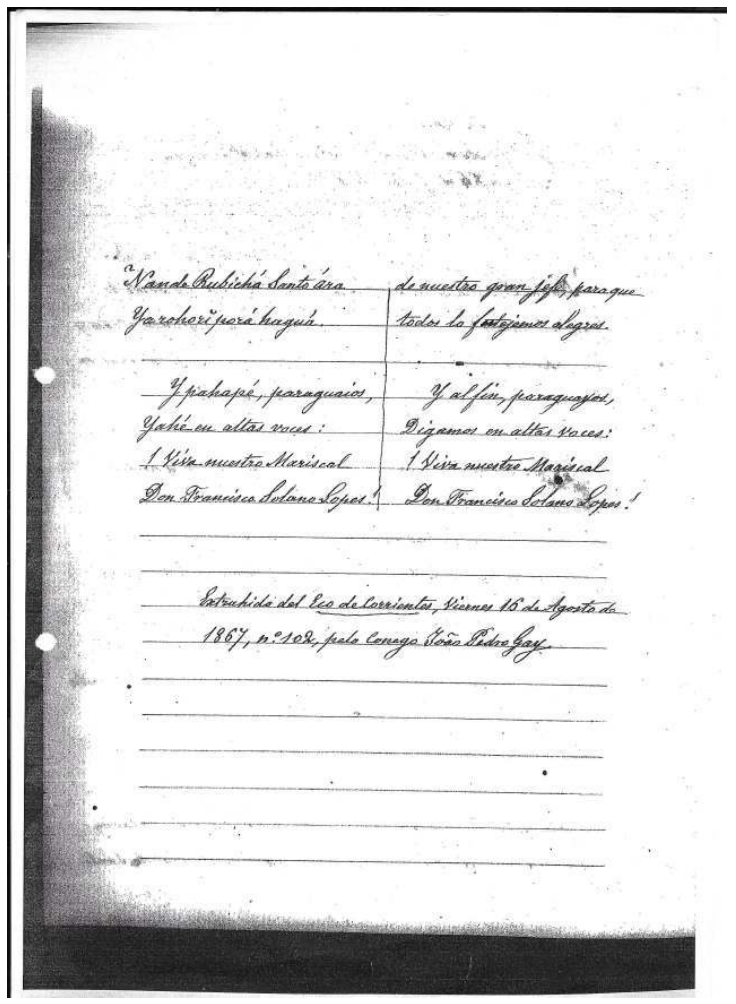

Imagen $\mathrm{N}^{\circ} 2$

Para entender la aparición del texto en la prensa correntina es necesario indagar el panorama político, social e ideológico de la provincia en torno a la Guerra de la Triple Alianza. Se sabe que en el orden nacional la guerra había suscitado resistencias y cuestionamientos, sobre todo por la alianza con Brasil, que había recibido críticas desde diferentes sectores incluido el propio partido de Mitre. En Corrientes las reacciones incluyeron también el apoyo directo a la causa paraguaya por parte de un sector de la sociedad. Este sector constituyó principalmente la facción que había sido derrotada en la batalla de Pavón (1861), acontecimiento decisivo a nivel nacional que había llevado a Mitre a la presidencia y que significó la derrota del partido federal y el principio de la hegemonía de Buenos Aires sobre el resto del país. La batalla de Pavón tuvo consecuencias también en la política provincial pues llevó a la gobernación al liberal Manuel Ignacio Lagraña, quien gobernaría a favor del gobierno central. En abril de 1865 el Paraguay invade Corrientes, obligando al gobierno de Lagraña a retirarse al campo. Esta situación reavivó el espíritu de oposición política entre la antigua provincia litoraleña y Buenos Aires (Ramírez Braschi 2014, pp. 74-76). ${ }^{18}$ El gobierno provincial fue sustituido por un triunvirato formado por vecinos de Corrientes que respondían a Asunción. Hubo una cooperación explícita entre sectores de la elite provincial y las fuerzas de ocupación paraguayas. Estas acciones se explican no solo por la situación política provincial, sino también por la existencia de lazos familiares, comerciales y culturales que unían desde tiempos coloniales al país guaraní con la provincia argentina. El "paraguayismo" correntino se expresó incluso en batallones provinciales que lucharon junto a los paraguayos, y en una bandera provincial que sustituyó a la argentina durante la ocupación y que se izaba junto a la paraguaya (Ramírez Braschi 2004, p. 56; Ramírez Braschi 2014, p. 76). 
19 Si bien el diario Eco de Corrientes aparece en época posterior a la ocupación paraguaya, su edición está vinculada al gobierno de Evaristo López, un federal antiporteño que sucedió a Lagraña llegando a la gobernación a principios de 1866. Eco de Corrientes fue evidentemente un instrumento oficialista "fundado para contrarrestar la prédica de $E l$ Nacionalista, estando al servicio de Don Evaristo López” (Méndez Paz 2007 [1953], p. 39). ${ }^{19}$ Por otra parte Eco de Corrientes fue financiado con fondos oficiales, trabajaron en él funcionarios del gobierno de Evaristo López, y dejó de aparecer poco antes de que este abandonara el gobierno por causa de las presiones de los liberales. En este sentido la aparición de nuestro poema en este medio no sorprende pues responde a intenciones de este sector de la sociedad provincial, el que apoyó al Paraguay y buscó confrontar con la ideología del gobierno de Buenos Aires.

Lejos de ser un mero panfleto propagandístico, Eco de Corrientes fue también una tribuna de opiniones en donde se publicaron además textos contra el Mariscal López, elogios al ejército aliado, e incluso poemas en portugués vitoreando a Don Pedro II (cf. $\mathrm{n}^{\circ} 71$, 10.05.1867), aunque no faltan notas de marcada simpatía con el Paraguay (cf. $\mathrm{n}^{\circ} 123$, 1.11.1867). Esta heterodoxia se explica pues el diario reservó un espacio para publicar textos de particulares "a precios convencionales", como anuncia su frontispicio. Posiblemente nuestro poema responda a una de estas publicaciones que vecinos de la elite provincial pagaban para impactar en la opinión pública o dar un alcance popular a ciertas ideas políticas. Tengamos en cuenta que en esta época el periodismo jugó un rol importante en los debates ideológicos y en las opiniones en torno de la guerra, y tuvo como uno de sus ejes centrales el tema de la identidad nacional (Baratta 2013, pp. 79-80). En este contexto un poema dedicado al Mariscal López involucra una confrontación directa con los sectores que apoyaron la guerra y al gobierno central, y un intento de reafirmar la solidaridad mutua entre los correntinos vinculados al proceso de colaboración con el Paraguay, algunos de los cuales gobernaban entonces la provincia.

\section{El contexto sociolingüístico y las características del poema}

21 Más allá de su tema, es especialmente la lengua del poema la que hace que el mismo adquiera un valor simbólico específico. De hecho se trata de la lengua que en esta época es común a paraguayos y correntinos (Zajícová 2009, pp. 30-34). No es aventurado postular que la guerra habría puesto de relieve una relación directa entre identidades nacionales y lingüísticas. La presencia de brasileños en el territorio correntino (donde estaban instaladas las provisiones y los hospitales de su ejército) habría tenido como consecuencia una mayor visibilidad de la lengua portuguesa. ${ }^{20}$ Por contraste, el castellano tuvo que haber servido como marca de identidad de las tropas argentinas. A su vez, en este mismo contexto, el guaraní se volvía símbolo de la identidad nacional paraguaya (Lustig 1997; Zajícová 2009, p. 34). En este sentido el poema en guaraní evoca, al publicarse en Corrientes, una relación indisociable entre esta provincia y el Paraguay, apelando a la lengua como medium de esta relación, evocando los lazos de identidad cultural, histórica y lingüística entre la provincia argentina y el país vecino.

Existen otros detalles también significativos. El lector habrá notado que el poema está escrito en una variedad híbrida entre español y lengua indígena, es decir, no se trata de un guaraní "puro", sino de una lengua que acusa fenómenos de contacto lingüístico como 
son los préstamos léxicos (p. ej. santo, el ataque), gramaticales (porque, artículos) y hasta una instancia de code switching en el final (¡Viva nuestro Mariscal / Don Franciso Solano López! ). Esta variedad mixta de guaraní -en Paraguay llamada yopará o jopara- no es sin embargo artificial sino que responde a la lengua de uso coloquial de la sociedad de la época. ${ }^{21}$ Se trata de la misma variedad lingüística de guaraní que en Paraguay alcanza, en estos años, por primera vez un estatuto semioficial y es llevada a la escritura. En efecto, en el seno del periodismo de guerra paraguayo, si bien se debatió una idea de purismo, se consideró como modelo el habla ya bastante hispanizada del campesinado que combatía en las trincheras (Lustig 2006, pp. 253-254). Los periódicos de guerra paraguayos contribuyeron en este sentido a prestigiar el guaraní mezclado. En lo que concierne a Corrientes, nos parece que la reproducción del poema sin mayores cambios constituye un indicio de que en la época hubo una predisposición a aceptar este guaraní popular para el uso escrito. Es posible que la publicación del texto esté reflejando cierto entusiasmo por la escritura y difusión de esta variedad del guaraní en letra de imprenta, experiencia que llegaba desde el Paraguay y que habría despertado el interés de los sectores paraguayistas de la provincia. Esta actitud constituye la cara opuesta del rumbo que tomará la política lingüística en Corrientes una vez afirmada la ideología liberal tras la Guerra Grande. Esta ideología, que será la dominante en el siglo XX, está marcada por la homogeneización lingüística, el purismo y la tendencia normatizante, y será contraria no solo al uso de la lengua indígena sino también a aceptar la variedad "mezclada" (cf. Cerno 2013, p. 38; Cerno 2017, en prensa). Pese a todo debe tenerse en cuenta que Eco de Corrientes no publicó otros poemas en guaraní, al menos esto se infiere de las colecciones consultadas (cf. nota 15), y el poema que copió Gay parece haber sido una experiencia aislada. Esperamos que futuras investigaciones nos contradigan en este punto.

Un aspecto en el que difiere nuestro poema de la versión publicada en el Paraguay es el de la ortografía. Este hecho supone una toma de posición respecto de las tradiciones gráficas vigentes en aquella época. El guaraní había recibido una primera estandarización gráfica por parte de los jesuitas y franciscanos a principios del siglo XVII, pero los periódicos de guerra paraguayos hacen tabula rasa e inventan su propio sistema ortográfico. Para comparar los textos desde esta perspectiva presentamos ahora la versión paraguaya (véase IMAGEN N ${ }^{\circ}$ ):

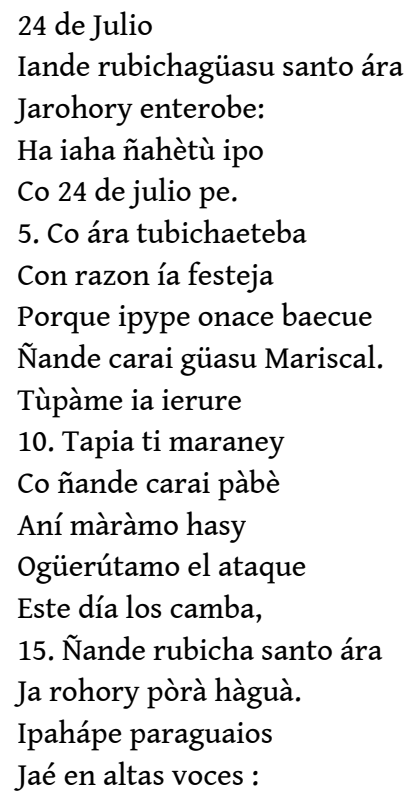


!Viva nuestro Mariscal

20. D. Francisco S. Lopez!

(Extraido de El Cabichuí. Año 1, n²2, p. 4, cf. Escobar y Salerno 2016)

Silvia2017-06-29T01:29:00SFalta insertar imagen $\mathrm{N}^{\circ} 3$

Imagen $\mathrm{N}^{\circ} 3$

En efecto, acaso por la falta de tipos de imprenta especiales, pero también por una reflexión sobre los valores fonéticos de la lengua, los periódicos paraguayos desarrollaron un sistema ortográfico a partir de la llamada "Convención de Paso Pucú" (Lustig 2006, p. 249 y ss. y nota 31). Para las vocales nasales del guaraní, mientras que el alfabeto jesuítico apeló al acento breve del sistema latino (Â, Ê, Î, ĩ ô, Û), el alfabeto de Paso Pucú se valió del acento grave (à, è, ì, ỳ, ò, ù). Una rápida comparación entre ambas versiones pone de manifiesto que, mientras el texto correntino, si hemos de confiar en la transcripción de Gay (y no hay motivos para dudar de ella), continúa con el alfabeto jesuítico, el poema paraguayo constituye una ruptura. Ejemplos concretos son TupÂ, mÂrÂ vs. Tùpà, màrà, en los versos 9 y 12 de cada uno de los textos. Otro aspecto constituye la representación de la "sexta vocal" del guaraní, la /i/, que el alfabeto jesuítico simbolizó con los grafemas <ì e $<\breve{y}>$, y el alfabeto paraguayo sólo con $<\mathrm{j}>$, diferencia que vemos por ejemplo en haçı̌ vs. hasy, verso 2; yarohorĭ vs. iarohory, verso 16. La fricativa sorda /s/ del guaraní, escrita $\langle\mathrm{s}>$ en el texto paraguayo se representa en el poema correntino con $\langle$ <̧>, rasgo también típico del alfabeto jesuítico (ej.: guaçú, haçı̆, vs. güasú, hasy, versos 1 y 12 respectivamente). La consonante guaraní /gw/ es representada con <gü> en el sistema paraguayo pero <gu> en el texto correntino (güasú, ogüerú vs. guaçú, oguerú, versos 1 y 13). Otro rasgo divergente es la representación <i> tanto para la vocal alta /i/ (ipo, verso 3) como para la semiconsonante / $\mathrm{j}$ / en el poema paraguayo (iaha, iaierure, versos 3 y 9 ), hecho que en el poema correntino, siguiendo el alfabeto jesuítico, corresponde $\mathrm{a}<\mathrm{y}>$ (yaha, yayeruré).

Tenemos así la coexistencia de dos sistemas gráficos, uno innovador, el paraguayo, y otro más conservador, el jesuítico, cuyos últimos escritores habían ya desaparecido (cf. Thun 2003, p. 10-11) y que ahora es retomado en Corrientes. Es posible que para explicar esta diferencia tengamos que apelar a la percepción del público al que apuntaban los medios gráficos, tanto en Paraguay como en la provincia argentina. Así, parecería que los editores de Eco de Corrientes apelaron a lectores de los que suponían estaban familiarizados o al menos conocían el alfabeto jesuítico y podían interpretar de esta manera mejor el texto. Este sector correspondía a la clase letrada y culta, clase que posiblemente no fue ajena a la circulación de textos escritos en guaraní reduccional posterior a la expulsión de los ignacianos. Tengamos en cuenta también que el alfabeto jesuítico tuvo alguna presencia en la administración provincial hasta al menos la década de 1830. En efecto, por esos años ubicamos las últimas actas de cabildo escritas por indígenas de las ex reducciones que habían fundado pueblos nuevos cerca del estero Iberá. ${ }^{22}$ Por su parte, el sistema gráfico creado en el Paraguay había sido ideado más bien para la reproducción oral antes que para la lectura directa (Lustig 2006, p. 252). En este sentido no se habría pensado tanto en lectores concretos, sino en lecturas públicas donde se reproducían oralmente los textos para un auditorio conformado principalmente por soldados analfabetos. Si bien esto se aplica sobre todo al caso del único medio de prensa completamente escrito en guaraní, el Cacique Lambaré, el mismo alfabeto rigió para todos los periódicos de guerra paraguayos incluyendo el Cabichuí. que podrían estar anunciando la percepción de diferencias entre el guaraní del Paraguay 
y el guaraní de Corrientes. Se encuentran hechos de variación, por ejemplo el intensificador ete en el poema paraguayo corresponde a ite en el texto correntino (tubicha eteba vs. tubichaiteba, verso 5). Otra variante involucra al rasgo de la armonía nasal, por ejemplo iande (verso 1) en el poema paraguayo, correspondiente a ñande que muestra nasalización completa. No obstante en el poema correntino la representación oral de palabras nasales es más marcada, y hallamos escritas con acento oral porá, haguá, ñahetú (versos $16 \mathrm{y}$ 3), correspondientes a pòrà, hàguà, ñàhetù, en el texto paraguayo. ${ }^{23}$ Otro ejemplo de variación es el uso raro del verbo yahé "decimos" en el verso 18, cuya forma tradicional paraguaya y jesuítica es yaé (pronunciado [dja'Pe]). No encontramos registros de la forma yahé en nuestros materiales, pero la expresión no puede atribuirse simplemente a un descuido del copista o del editor. Este cambio de fonema glotal / $/ \mathrm{p}$ por la aspirada /h/ es común en la típica expresión correntina aikuaha [ajkwa'ha] 'conozco', dicha en guaraní paraguayo aikuaa [ajkwa' $\mathrm{Pa}$ ]. Otras expresiones no son variantes sino palabras diferentes en ambos poemas. Es el caso de mârâmi vs. maraney en el verso 10, y de peabé vs. pàbè en el texto correntino y paraguayo respectivamente.

\section{Conclusiones}

Nuestra exploración de la figura de João Pedro Gay en tanto investigador nos lleva a postular que el mismo tuvo un interés especial por las lenguas de la región, especialmente por el guaraní, hecho que justificamos con la presentación de un texto copiado por su mano y que a su turno constituye una importante contribución a nuestra visión actual del pasado sociolingüístico de Corrientes. Nuestra investigación muestra también, en el plano de la biografía del padre Gay, que su legado merece mayor atención tanto por sus observaciones del contexto como por la posibilidad de ofrecer otras fuentes de datos novedosas. Por el lado del poema que analizamos, mostramos que hubo hacia mediados de siglo XIX la conciencia de una identidad lingüística común en la región guaranítica, y que la misma tuvo connotaciones políticas vinculadas a las ideologías dominantes. El análisis del poema muestra también que por la misma época hubo diferencias entre el Paraguay y Corrientes con respecto a la lengua, no solo en cuanto al sistema ortográfico usado sino también por el uso que se dio al guaraní escrito en los medios de prensa y a sus implicancias en torno a la relación entre identidad nacional y lengua. Por otra parte la transcripción de Gay del poema permite aproximarnos por primera vez a algunas diferencias dialectales que pudieron haber existido en el guaraní en ambas regiones y que se confirman parcialmente en las variantes actuales. Para concluir queremos subrayar que un examen más detenido del legado lingüístico de Gay, posiblemente más grande del que conocemos, podría realizar en el futuro mayores contribuciones.

\section{BIBLIOGRAPHY}

Acosta, M. A. (2016). Ad majorem dei gloriam - Espace de dieu et domaine des hommes, analyse des missions jesuites du paracuaria $(1610-1767)$ a partir de l'archeologie phenomenologique, cas 
d'étude Nuestra Señora de Loreto (i et ii) et San Ignacio Mini (i et ii), Tesis doctoral, Université Laval, Québec, 2016. [On line]. Dispoinble: http://www.theses. ulaval.ca/ 2016/32795/32795.pdf. Avé-Lallemant, R. (1859). Reise durch Süd-Brasilien im Jahre 1858. 2 Tomos. Leipzig: Brockhaus. Trad. portugesa: Viagem pelo sul do Brasil no ano de 1858, trad. del Instituto Nacional do Livro [por Teodoro Cabra], Rio de Janeiro: Ministério da Educação e Cultura, 1953.

Baratta, M. V. (2013). El litoral y la batalla de la pluma. La identidad nacional argentina en los periódicos de Entre Ríos y Corrientes durante la Guerra del Paraguay. Folia Histórica del Nordeste, 21, 75-96.

Bell, S. (2010). A life in shadow. Aimé Bonpland in Southern South America, 1817-1858. Stanford: California University Press.

Blake, A. V. A. S. (1883-1902). Diccionario Bibliographico Brazileiro, 6 tomos, Río de Janeiro: Typographia Nacional.

Cerno, L. (2013). El guaraní correntino. Fonología, gramática, textos. Frankfurt am Main: Peter Lang.

Cerno, L. (2017, en prensa). Aspects of Dialectal Diversification of Guarani in Paraguay and Corrientes: Contact between Two Given Languages in Different Settings. En B.Estigarribia y J. Pinta (Eds.). Guaraní Linguistics in the $21^{\text {st }}$ Century. Leiden: Brill Verlag.

Cerruti, C. (2012). L'américanisme en construction. Une pré-histoire de la discipline d'après l'expérience du naturaliste Aimé Bonpland (1773-1858). Tesis doctoral, La Rochelle: Université de La Rochelle. [On line]. Disponible : http://www.tel.archives-ouvertes. fr/file/index/ docid/921277/ filename/2012Cerruti33829.pdf.

Cortés Conde, R. (1977). Latin America, a guide to economic history, 1830 - 1930. Berkeley: University of California Press.

D’Angelis, W. da R. (2003). o primeiro século de registro da língua kaingang (1842-1950), valor e uso da documentação etnográfica. [Ponencia presentada en el] $3^{\circ}$ Encontro Macro Jê, LALI-UnB, Brasilia, 3-6, diciembre de 2003. [On line]. Disponible: //www. portalkaingang.org/Primeiros 100anos.pdf

D’Angelis, W. da R. (2012). o Petit Vocabulaire Kaingang do Cônego Gay, manuscrito do IHGB. En: R. de S. Amado, (Org.), Estudos em línguas e culturas Macro-Jê (pp. 93-111). San Pablo: Paulistana.

D'Angelis, W. da R., Cunha, C. y Rodrigues, A. (Eds.). (2002). Bibliografia das línguas Macro-Jê, Campinas: IEL/Unicam. [On line]. Disponible: http://biblio.etnolinguistica.org /dangelis-2002bibliografia

Demersay, A. (1860-1865). Histoire physique, économique et politique du Paraguay et des établissements des Jésuites, Ouvrage accompagné d'un atlas de pièces justificatives et d'une bibliographie, 2 tomos y un Atlas. París: Hachette.

Escobar, T. y Salerno, O. (Comp.). (2016). Cabichuí. Periódico de la Guerra de la Triple Alianza. 2da edición corregida y ampliada. Asunción: Biblioteca Nacional del Paraguay / Secretaría Nacional de Cultura.

Furlong, G. (1953). Historia y bibliografía de las primeras imprentas rioplatenses, 1700 - 1850: misiones del Paraguay, Argentina, Uruguay. Buenos Aires: Guarania.

Gay, J. P. (1942 [1863]). História da República Jesuítica do Paraguay. Río de Janeiro: Imprensa Nacional. $1^{\circ}$ ed. en Río de Janeiro: Typ. de Domingos Luiz dos Santos, 1863. [On line] Disponible: http://www.ihgb.org.br/publicacoes/revista-ihgb/itemlist/filter.html? category=9\& moduleId $=147$ 
Gay, J. P. (1867). Invasão paraguaya na fronteira brazileira do Uruguay: desde seu principio até seu fim (de 10 de junho a 18 de setembro de 1865. Río de Janeiro: Typographia imperial e constitucional de J. Villeneuve \& C.

Hamy, E. T. (1906). Aimé Bonpland, médecin et naturaliste, explorateur de l'Amérique du Sud; sa vie, son œuvre, sa correspondance; avec un choix de pièces relatives à sa biographie. París: $\mathrm{E}$. Guilmoto. [On line]. Disponible : http://www.archive.org/details/ aimbonplandm0ohamy

Lustig, W. (1997). Chácore purahéi - Canciones de guerra. Literatura popular en guaraní e identidad nacional en el Paraguay. Ñemitỹ, 35, 21-36. [On line]. Disponible: http://www.staff.unimainz.de/lustig/guarani/chacpura/chactext.htm

Lustig, W. (2006). La lengua del “Cacique Lambaré” (1867), primer modelo de un guaraní liteario. En W. Dietrich y H. Symeonidis (Eds.). Guaraní y "Mawetí-Tupí-Guaraní”. Estudios históricos y descriptivos sobre una familia lingüística de América del Sur (pp. 241-258). Münster: Lit Verlag.

Maestri, M. (2012). o singular relato do cônego João Pedro Gay sobre a invasão paraguaia da Província de São Pedro do Rio Grande do Sul. Estudios históricos, Centro de Documentación Histórica del Rio de la Plata y Brasil DHRPyB, Año IV, diciembre 2012. [On line]. Disponible: http://www.estudioshistoricos.org/edicion9/eh0909.pdf.

Mantilla, M. (1887). La Bibliografia Periodistica de La Provincia de Corrientes. Buenos Aires: Imprenta y Librería de Mayo.

Méndez Paz, E. (2007 [1953]). Periódicos Correntinos. 1825-1900. 2a edición. Instituto de Investigaciones Históricas y Culturales de la Provincia de Corrientes. Corrientes: Amerindia.

Moussy, V. M. (1859). Memoria histórica sobre la decadencia y ruina de las misiones jesuíticas en el seno del Plata. Su estado en 1856. Paraná: Imprenta del "Nacional Argentino".

Moussy, V. M. (1860). Notice sur la vie de M. Bonpland en Amérique, Plata, Paraguay et Missions. Bulletin de la Société de géographie de Paris, $4^{\circ}$ serie, t. XIX, Enero-Junio1860.

Moussy, V. M. (2005 [1860-1864]). Descripción geográfica y estadística de la Confederación Argentina. Trad. española, ed. a cargo de Beatriz Bosch. Buenos Aires: Academia Nacional de la Historia/Dunken, 3 tomos. Editado por primera vez en París: Firmin Didot, Volumen 1, 2 (1860), Volumen 3 (1864) con Atlas (1869).

Nogueira, B. C. de A. (1879). Manuscripto Guarani sôbre a primitiva catechese dos Indios das Missões. Obra composta em castelhano pelo p. Antonio Ruiz Montoya, vertida para guarani por outro jesuita, e agora publicada com a traducção portugueza, notas, e um esbôço grammatical do abanheem pelo Dr. Baptista Caetano de Almeida Nogueira. Annaes da Bibliotheca Nacional do Rio de Janeiro, vol. 6, Fasc. 1. Rio de Janeiro: Typ. G. Leuzinger \& Filhos. [On line]. Disponible: http:// www.etnolinguistica.org/biblio: nogueira-1879-manuscripto.

Obermeier, F. (2007). Robert Avé-Lallemant (1812-1884) und seine Brasilienbücher. Jahrbuch Martius-Staden, 54, 221-240. [On line]. Disponible: http://www.macau.uni-kiel.de.

Ramírez Braschi, D. (2004). La Guerra de la Triple Alianza a través de los periódicos correntinos. 1865-1870. Corrientes: Moglia Ediciones.

Ramírez Braschi, D. (2014). La Guerra del Paraguay en la provincia de Corrientes. Impactos políticos, daños y consecuencias en la población civil. Corrientes: Moglia Ediciones.

Rodrigues, J. H. (1954). Padre Gay. Província de São Pedro, Porto Alegre. 19, 75-93.

Teixeira Weber, B. y Oliveira da Silva, J. (2012) Padre Gay: um cônego ilustrado na campanha gaúcha. Estudios iberoamericanos, V. 38, 2012, no. 1, 144-160. [On line]. Disponible: http:// 
www.revistaseletronicas.pucrs.br/ojs/index.php/iberoamericana/article /

download/11591/8015.

Thun, H. (2003). Evolución de la escripturalidad entre los indígenas guaraníes. En E. Ridruejo y M. Fuentes (Coord.), I Simposio Antonio Tovar sobre Lenguas Amerindias. Instituto Interuniversitario de Estudios de Iberoamérica y Portugal. Valladolid: Universidad de Valladolid, 9-23.

Zajícová, L. (2009). El bilingüismo paraguayo. Usos y actitudes hacia el guaraní y el castellano. Frankfurt/Madrid: Vervuert Iberoamericana.

\section{NOTES}

1. Para su biografía véase también Rodrigues 1954, Maestri 2012, Blake 1883-1902, Teixeira Weber y Oliveira da Silva 2012.

2. Publicada por primera vez en la Revista do Instituto Histórico e Geográfico Brasileiro, tomo XXVI, 1863, pp. 5-120, 183-268, 351-448, 589-838. Para la primera edición en forma de libro cf. Gay 1942 [1863]

3. Ver Guillermo Furlong, 1953, pp. 92-93 y nota 78. Cf. el comentario de Marcelo Alejandro Acosta, "L'un des premiers travaux à avoir analysé l'exode du Guairá et qui est communément employé pour décrire ce moment, a été écrit par João Pedro Gay, vicaire de la mission de San Juan Borja qui a publié «História da República do Paraguai» (1863) a répandu des affirmations sans base historique ni documentaire, lesquelles ont été répétées par la suite par des auteurs contemporains. Parmi ces erreurs, on a trouvé les dates des fondations des réductions primitives de San Ignacio Miní I et Loreto I. Cet auteur indique que les deux réductions ont été fondées en 1555 par le conquéreur espagnol Ñuflo de Chavez $(1518$ - 1568) et originellement furent des villages espagnols, qui par la suite, furent administrés par la Compagnie de Jésus. Cela a découlé de l'action évangélisatrice de l'ordre dans des espaces de frontières «Si bien que les villages de Loreto, de Santiago et Santa Maria de Fe ont été des villages espagnols, mais leur direction a été confiée aux Jésuites»", Acosta 2016, p. 285.

4. Cf. Gay 1867 en la bibliografía.

5. El bibliotecario Ramiz Galvão menciona, en su introducción a la edición de Almeida Nogueira de Conquista Espiritual, la circunstancia de haber Gay trabajado con uno de los manuscritos conocidos de la traducción guaraní a la obra de Montoya. Cf. Nogueira 1879, pp. VIII-IX. Para el interés de Gay en el guaraní jesuítico cf. Gay 1863, p. 185 y ss., pp. 186-240, pp. 241-268 y notas. Fue también Gay quien por primera vez identificó al probable autor del manuscrito guaraní de “Conquista Espiritual" que llegó a sus manos. Basándose en las iniciales, determinó que se trataba del jesuita Jaime Bonenti, ver Nogueira 1879, pp. VIII-IX.

6. IHGB Signatura DL 1116.6. Véase D’Angelis, W. da R. (2012) para una descripción del texto.

7. Ver también D'Angelis, W. da R., Cunha, C. y Rodrigues, A. (Eds.) 2002. El legado de Gay como lingüista podría ser no obstante mayor. Parte de su biblioteca, donde se cuentan los libros que legó a Pedro II entre otros documentos, consta hoy en la Biblioteca Nacional de Río de Janeiro. En ella hallamos el documento que aquí presentamos. Pensamos que es necesaria una investigación más detenida de esos papeles.

8. Sobre el contacto Gay-Bonpland ver Bell 2010. Sobre sus colecciones, Bell 2010, pp. 8-10, sobre documentos que Gay escribió para el ministerio francés ver Bell 2010, p. 235, nota 32. Los documentos más importantes constan en "Ministre de l'intérieur, Paris [sic] 15.08.1859 F, 3974, fol 48, Archives nationales de France", manuscrito, las primeras 16 páginas datadas en San Borja el 29.08.1858 con postscriptum sobre la colección de Bonpland.

9. Publicada en Didot, París, en 1835. 
10. Cf. Cerruti 2012, p. 559.

11. Ver Moussy 1859.

12. Ver Moussy 2005 [1860-1864].

13. Hamy se refiere también a Martin de Moussy (1860).

14. Avé-Lallemant 1859. Sobre otros de sus libros y su importancia para la discusión sobre la emigración alemana en el Brasil ver Obermeier 2007.

15. Nos referimos a las colecciones de la Biblioteca Pública de la Universidad Nacional de la Plata y del Archivo General de Corrientes, que hemos consultado.

16. Los datos del catálogo son los siguientes: Autoridade: LOPES, Francisco Solano Título: Hymno en Guarany, festejando os anos do Marechal F.S.Lopes Local: S.l. Data: 24 jul. 1867 Paginação: 1 doc. 2 p. Coleção: Decimal Notas: Original. Extraído do Eco de Corriente, Viernes, 16 de agosto de 1867, nº102, pelo cônego João Pedro Gay.

17. Sobre su frecuencia de aparición nos dice Cortés Conde que fue "quincenal y nivel provincial" (Cortés Conde 1977, p. 130). Méndez Paz dice que "apareció dos veces por semana, el n 1 apareció el 24 de agosto de 1866 y el último, el 186, el 26 de marzo de 1868" (Méndez Paz 2007 [1953], p. 39-40). Por nuestra parte verificamos los datos de este último autor con la excepción de que el diario apareció en realidad hasta el 26 de mayo de 1868.

18. "La ocupación paraguaya y la consecuente elección de una Junta Gubernativa integrada por correntinos y filialmente ligada al gobierno de Asunción, dividirá literalmente a Corrientes. La sociedad política tomará partido por los invasores o por el Gobierno de Lagraña. No habrá intermedio en esta cuestión". (Ramírez Braschi 2014, p. 25)

19. La "prédica" a la que alude la cita constituye la insistencia con que aquel medio pedía la condena de ciertos vecinos y funcionarios por traición a la patria o colaboracionismo durante la ocupación paraguaya (Ramírez Braschi 2004, p. 74)

20. Que fue la lengua de muchas notas y textos poéticos publicados en Eco de Corrientes, y encargadas seguramente por el gobierno imperial (cf. por ejemplo Eco de Corrientes, $n^{\circ} 19, n^{\circ} 21, n$ - 35, de 1866)

21. Sabemos que Paraguay y Corrientes formaban parte de una misma área lingüística que hasta bien entrado el siglo XIX se caracterizó por amplia difusión del guaraní y escasa presencia del español, lengua esta última casi exclusiva de los usos oficiales, o reservada para hablar con extranjeros (Zajícová 2009, pp. 30-34). Diversos testimonios de viajeros muestran que había una percepción unificada sobre la región en su aspecto lingüístico. John Parish Roberson describe que hacia 1838 "aquí [Asunción] lo mismo que en Corrientes, los hombres hablan poco y con cierta resistencia el español” (cit. en Zajícová 2009, pp. 32). No obstante, esta área lingüística nunca había estado libre de la influencia permanente del español, como sí lo habían estado las Reducciones Jesuíticas, y ya hacia mediados del siglo XVIII fue evidente la distinción entre un guaraní "puro" hablado en los pueblos jesuíticos y un guaraní mezclado propio de los pueblos españoles (Zajícová 2009, pp. 69-71, Cerno 2013, p. 31)

22. Se trata de los pueblos de San Miguel y Loreto, cuyas actas de fundación en 1827 se redactaron en guaraní. El alfabeto jesuítico era el único que estos indígenas conocían. Copias en español de estas cartas pueden verse en el Archivo General de Corrientes.

23. El guaraní cuenta con vocales orales y vocales nasales. Como la nasalidad depende parcialmente de la prosodia, se habla de un "acento oral" y de un "acento nasal" (acento que no tiene nada que ver con la "tilde" o el acento gráfico). Por su parte, estudios actuales indican la tendencia del guaraní correntino a oralizar vocales tradicionalmente nasales (Cf. Cerno 2013, p. 85 y ss.) 


\section{ABSTRACTS}

Our contribution highlights for the first time the French cleric João Pedro Gay (1815-1891) as a leading figure of Guaraní linguistic research in the nineteenth century. We are basing our analysis on a poem in Guarani copied by his hand from the local newspaper Eco de Corrientes in 1867. This poem is in itself a still unknown valuable source for XIX century Correntinean Guarani and represents a part of a larger legacy of Gay as researcher of the Guarani language. We contextualize the poem in its historical and sociolinguistic framework taking into account the specific situation in Argentine during the War of The Triple Alliance and its underlying linguistic ideologies. We conclude defining Gay's copy of the poem as part of an important linguistic contribution enabling us to acquire an outstanding and comprehensive knowledge about the history of Guarani varieties in this region.

Este trabajo destaca la figura del clérigo francés João Pedro Gay (1815-1891) como referente de la investigación lingüística del guaraní en el siglo XIX, tomando como base una copia de un poema en guaraní hecha por el propio clérigo en 1867. El poema, publicado en el diario Eco de Corrientes, constituye un antecedente del guaraní correntino del siglo XIX y representa a su vez una pequeña porción de lo que podría ser el legado de Gay como investigador e historiador del guaraní. En este sentido ofrecemos una interpretación del texto teniendo en cuenta el contexto histórico y sociolingüístico en que se enmarca, el cual incluye la Guerra de la Triple Alianza y las diferentes políticas lingüísticas y representaciones sociales que se desarrollaban en aquel tiempo en torno al guaraní. Por último concluimos valorando el documento de Gay como una importante contribución a nuestro conocimiento de la historia del guaraní y sus variedades en la región.

\section{INDEX}

Keywords: War of the Triple Alliance, history of linguistics in South America, Guarani language, language ideology, historical sociolinguistics

Palabras claves: Guerra de la Triple Alianza, historia de la lingüística sudamericana, lengua guaraní, ideologías lingüísticas, sociolingüística histórica

\section{AUTHORS}

\section{FRANZ OBERMEIER}

Universitätsbibliothek

Universidad de Kiel

Alemania

Correo electrónico: obermeier@ub.uni-kiel.de

\section{LEONARDO CERNO}

Universidad Nacional de Misiones, Instituto de Estudios Sociales y Humanos 
y Consejo Nacional de Investigaciones Científicas y Tecnológicas

Argentina

Correo electrónico: leonardo_cerno@yahoo.com.ar 